\title{
A Distinct Proof on Interplay between Trehalose and GdnHCl for the Stability of Stem Bromelain
}

\author{
Anjeeta Rani and Pannuru Venkatesu* \\ Department of Chemistry, University of Delhi, Delhi - 110 007, India
}

1. Figure S1: $\operatorname{Trp}$ fluorescence spectra of BM $25{ }^{\circ} \mathrm{C}$ : (a) in presence of varying concentrations of GdnHCl; 0.5 (red), 1.0 (green), 1.5 (blue), 2.0 (cyan), 3.0 (pink) and 4.0 M (yellow), (b) in presence of mixtures of $0.5 \mathrm{M}$ trehalose with varying concentrations of $\mathrm{GdnHCl} ; 0.5$ (red), 1.0 (green), 1.5 (blue), 2.0 (cyan), 3.0 (pink) and 4.0 M (yellow), (c) in presence of the mixtures of trehalose and $\mathrm{GdnHCl}$ at molar ratio of $1: 1$ i.e., $0.5 \mathrm{M}: 0.5 \mathrm{M}$ (red), $1.0 \mathrm{M}: 1.0 \mathrm{M}$ (green), 1.5 $\mathrm{M}: 1.5 \mathrm{M}$ (blue) and $2.0 \mathrm{M}: 2.0 \mathrm{M}$ (cyan) and (d) in presence of the mixtures of trehalose and $\mathrm{GdnHCl}$ at molar ratio of $1: 2$ i.e., $0.5 \mathrm{M}: 1.0 \mathrm{M}$ (red), $1.0 \mathrm{M}: 2.0 \mathrm{M}$ (green), $1.5 \mathrm{M}: 3.0 \mathrm{M}$ (blue) and 2.0 M : 4.0 M (cyan). The black spectra show BM in Buffer in all figures and Figs. are non-normalized Trp fluorescence spectra. Fig. S1(a) is taken from ref. [19]. (page S2)

2. Figure S2. Non normalized thermal transition curve of BM: (a) in presence of varying concentrations of GdnHCl; 0.5 (red), 1.0 (green), 1.5 (blue), 2.0 (cyan), 3.0 (pink) and $4.0 \mathrm{M}$ (yellow), (b) in presence of varying concentrations of trehalose; 0.5 (red), 1.0 (green), 1.5 (blue) and 2.0 M (cyan) and (c) in presence of mixtures of trehalose and $\mathrm{GdnHCl}$ at 1:1 molar ratio; $0.5 \mathrm{M}: 0.5 \mathrm{M}$ (red), 1.0 $\mathrm{M}: 1.0 \mathrm{M}$ (green), 1.5 M : 1.5 M (blue) and $2.0: 2.0 \mathrm{M}$ (cyan). (page S3) 


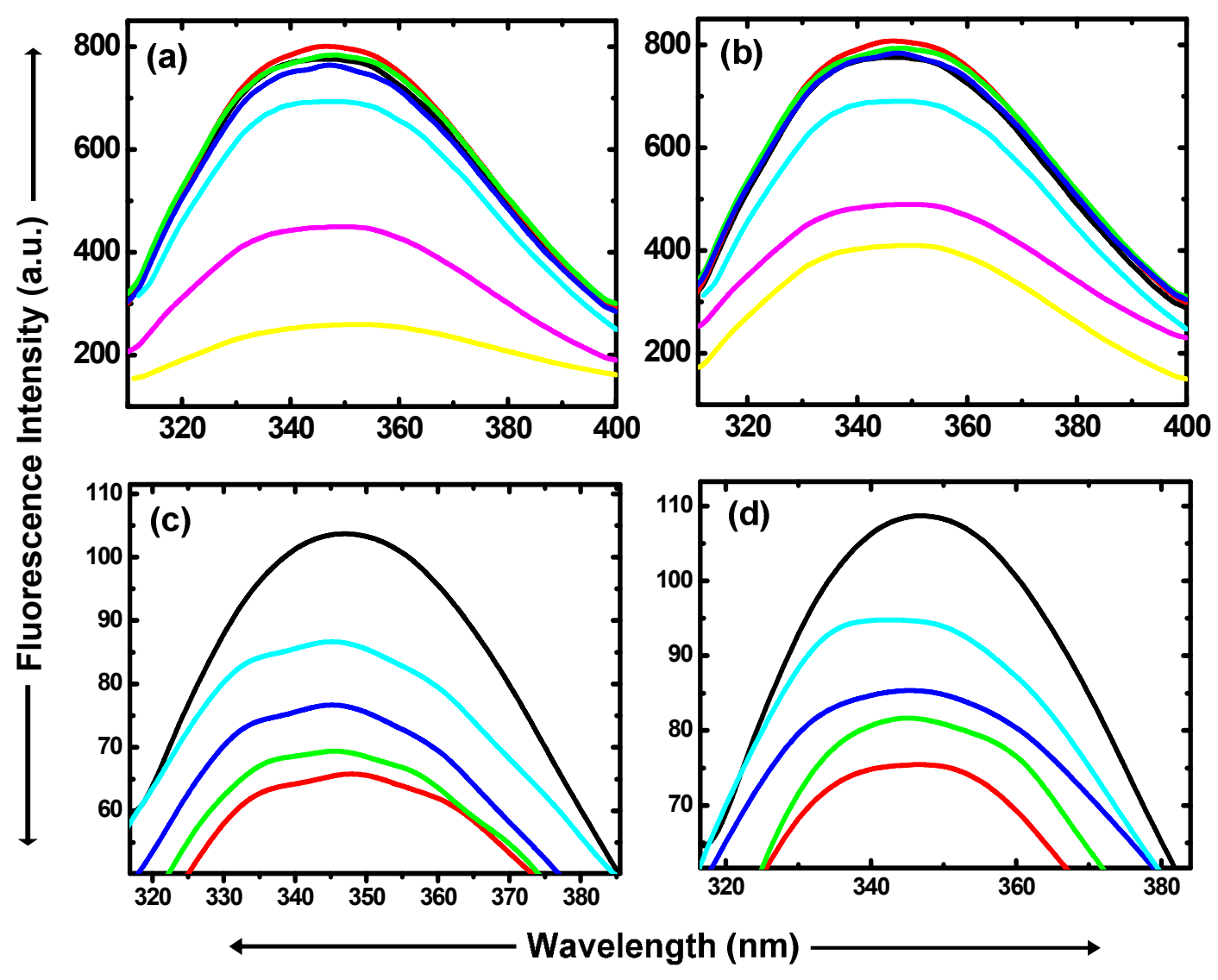

Figure S1. 

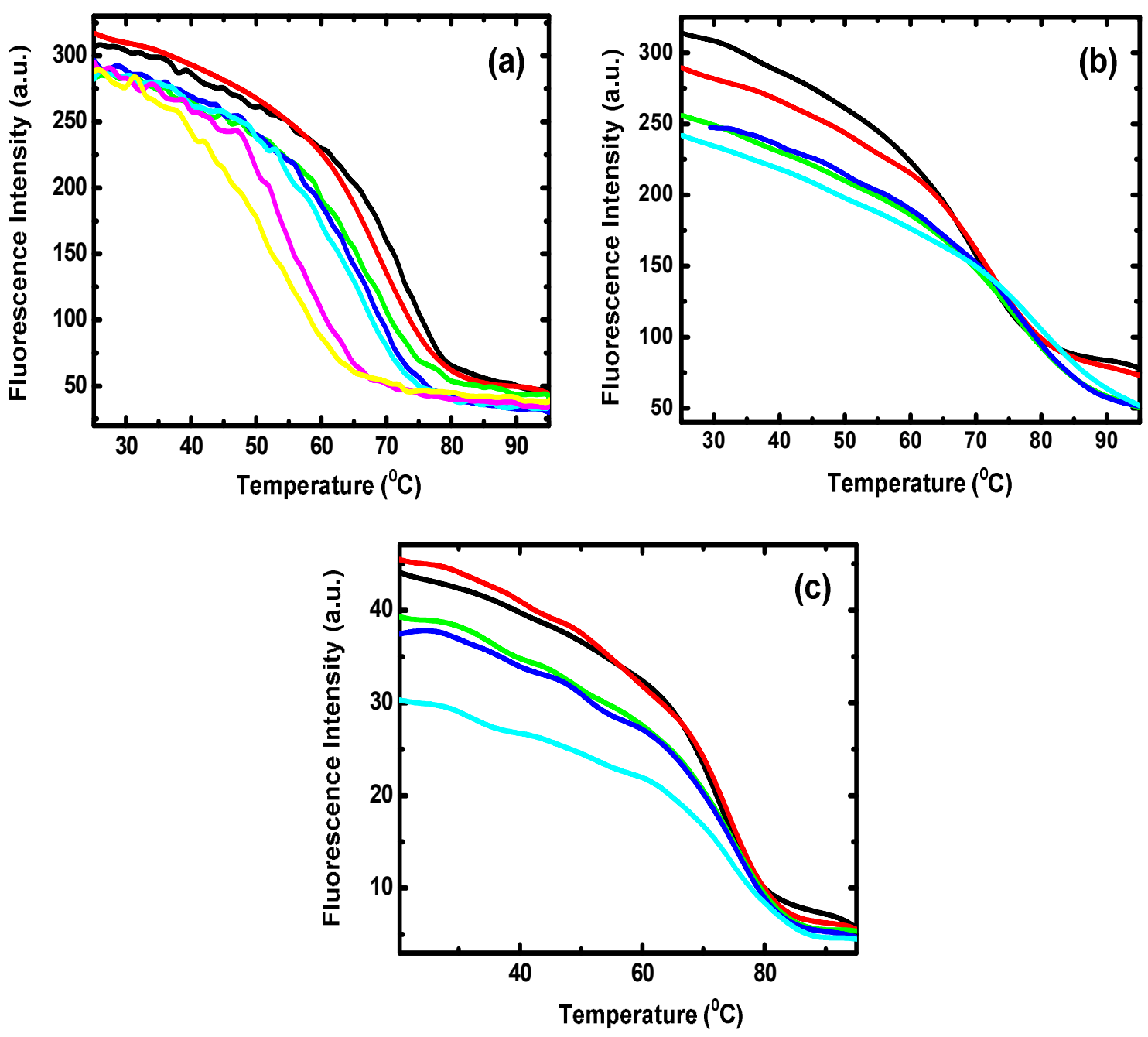

Figure S2. 